\title{
Diagnosis and management of pediatric tuberculosis in Canada
}

\author{
Ian Kitai MB BCh, Shaun K Morris MD MPH, Faisal Kordy MD, Ray Lam MN-ANP PHCNP
}

Cite as: CMAJ 2017 January 9;189:E11-6. doi: 10.1503/cmaj.151212

CMAJ Podcasts: author interview at https://soundcloud.com/cmajpodcasts/151212-view

$\mathbf{T}$ uberculosis (TB) is uncommon in children and adolescents in Canada, and diagnosis is often delayed because of a lack of diagnostic suspicion. ${ }^{1}$ Social determinants of health, including poverty, underscore its incidence and prevalence in countries with high TB burden but also among indigenous peoples in Canada in whom endemic TB remains a serious problem. We review the epidemiology, clinical presentation and diagnostic testing of children with TB in Canada and discuss safe management of latent TB infection. Because TB disease in childhood is best treated by an experienced multidisciplinary team, ${ }^{2,3}$ we discuss only briefly aspects of treatment relevant to a general clinician and refer to other resources for more details (Box 1).3,4

\section{Who gets TB in Canada?}

In Canada, TB is mainly a disease of foreign-born adults, who frequently acquire infection in their country of origin, and of Aboriginal Canadians. ${ }^{5}$ In 2014, 69\% of 1568 cases of TB reported in Canada were diagnosed in foreign-born patients and $21 \%$ were diagnosed in Canadian-born Aboriginal patients (Box 2). However, it is worth noting that those who are foreign born make up $22 \%$ of the Canadian population and those who are Aboriginal make up only $4 \% .{ }^{6}$ Although the overall annual rate of TB occurrence in Canada (4.4 per 100000 population) is much lower than the global rate of about 133 per 100000 , the national incidence rate obscures marked regional variability. In 2014, the rate of TB in Nunavut was estimated to be 227 per 100000 population - the highest rate in over a decade and one of the highest documented rates in the world. ${ }^{6}$

In 2014, less than $5 \%$ of all cases of TB in Canada occurred in children aged 14 years and younger. Rates of TB in children under 1, 1-4 and 5-14 years of age were 2.9, 2.0 and 0.8 cases per 100000 , respectively, and these rates have remained consistent over the past five years. Hospital-based data suggest that, although about $60 \%$ of non-Aboriginal children in Canada with TB were born elsewhere, most have at least one foreign-born parent; ${ }^{7}$ this mirrors the epidemiology of pediatric TB in the United States. ${ }^{8}$

Children born in Canada may acquire TB either from exposure within Canada, often to an infectious patient within the extended family, or when travelling abroad, frequently to visit family. ${ }^{7}$ Although most patients have an epidemiologic link, this may not be apparent

\section{KEY POINTS}

- Pediatric tuberculosis (TB) in Canada is mainly diagnosed in Aboriginal and foreign-born children, and children of foreign-born parents.

- About one-third of adolescents with TB in Canada have extrathoracic disease.

- Tuberculosis may mimic many conditions: specimens for TB culture are crucial for diagnosis.

- Best results are achieved if treatment is conducted by a team that includes physicians with experience in the management of pediatric TB.

initially. Occasionally, casual contact with a source case leads to disease in those without obvious risk factors. Diagnosis of TB in a young child should always prompt a search for an infectious source case.

\section{How is latent TB infection diagnosed?}

Latent TB infection refers to a small number of sequestered bacilli in the body that are controlled but not killed by the immune response. A diagnosis of latent TB infection is made if there is immunologic evidence of TB, no symptoms of TB, a physical examination not suggestive of TB disease and a chest radiograph that is either normal or shows evidence of old TB. ${ }^{9}$ Until 2001, the tuberculin skin test was the only widely available test for immunologic evidence of infection. ${ }^{10}$ Table 1 shows how to interpret the tuberculin skin test. ${ }^{10}$

In 2007, two interferon gamma release assays were licensed in Canada: QuantiFERON-TB Gold In-Tube test (Cellestis/Qiagen) and the T-SPOT.TB test (Oxford Immunotec). ${ }^{10}$ These blood tests use antigens present in Mycobacterium tuberculosis but not in bacille Calmette-Guérin vaccine and have better specificity, especially in patients immunized with this vaccine. Either the tuberculin skin test or interferon gamma release assay may be used for diagnosis, but the assays may be preferable in those who have received bacille Calmette-Guérin vaccine. ${ }^{11}$ Interferon gamma release assays are not recommended for testing in children younger than two years of age. ${ }^{11}$ The tuberculin skin test remains a good test for those with a 
high pretest probability of infection, such as close contact with infectious source cases. ${ }^{12}$

Tuberculosis disease implies an active process caused by M. tuberculosis that usually leads to signs, symptoms and/or radiologic features. Up to $20 \%$ of patients with TB disease have negative results for tuberculin skin tests and/or interferon gamma release assays. ${ }^{13,14}$

\section{How does TB disease present in children?}

In adult patients, pulmonary TB commonly presents with classic symptoms of fever, night sweats and weight loss. Pulmonary disease is common, and sputum samples are often positive upon acid-fast bacillus staining and/or culture positive. This is in contrast with the clinical features of pediatric TB, which are varied and often relate to the age at which the disease occurs (Box 2). Young children, especially those younger than five years of age, have difficulty containing TB infection to one organ system. Samples are usually paucibacillary, and there are frequent negative results for smears and cultures. Diagnosis is difficult. ${ }^{15}$

\section{Box 1: Evidence used in this review}

We identified primary literature, reviews and guidelines in PubMed from 1998 to 2015 using the following search terms: (1) "tuberculosis" and "pediatric," "paediatric," "children" or "adolescents;" and (2) "Canada" or one of the provinces or territories of Canada by name. We reviewed 288 abstracts and selected 57 for more detailed review. Separate searches were performed using term 1 and "symptoms" or "clinical presentation," and term 1 and "treatment." We also searched the Public Health Agency of Canada Web site for information about pediatric TB and TB epidemiology. Strong weight was given to the 2013 Canadian Tuberculosis Standards, ${ }^{3}$ which represents an evidence-based review and set of guidelines about epidemiology, diagnosis and management of TB in Canada, and to recent articles and systematic reviews. In general, treatment of pediatric TB has been evaluated using observational studies of poor or moderate quality.

\section{Box 2: Presentation of tuberculosis disease in children}

\section{Who is most likely to acquire TB in Canada?}

- Aboriginal children or children born outside of Canada

- Children with parents born outside of Canada

- Children who have travelled to countries endemic for TB, especially if they had a prolonged stay or contact with the local population

\section{When to suspect TB disease:}

- Epidemiology: as above; may not be apparent.

- Symptoms and signs (more common in older children and adolescents):

- Systemic ("B") symptoms: fever, weight loss, night sweats

- Pulmonary: productive cough, hemoptysis, chills

- Extrapulmonary: nonspecific, can present in any organ system

- Infants and young children commonly present with nonspecific signs and symptoms

Adapted from Paediatr Child Health 2015;20:83-8. ${ }^{4}$
TB disease will develop in about half of infants younger than 12 months who have TB infection, and serious forms of TB, including TB meningitis and miliary disease (a severe disseminated form), will develop in $10 \%-20 \%$ of these infants. ${ }^{16}$ Clinical features that suggest a diagnosis of TB in young children include a history of weight loss or failure to thrive (the equivalent of weight loss in the adolescent or adult), cough for more than two weeks and prolonged fever. ${ }^{17}$ However, these symptoms are not specific, and have poor specificity and sensitivity for diagnosis of pediatric TB in high TB-burden settings. ${ }^{18}$ Tuberculous meningitis has a subacute presentation that may begin with fever, listlessness and behaviour change but may evolve to coma and opisthotonus. ${ }^{19}$ Older children may complain of headache.

Primary infection in the older child usually produces a small pulmonary parenchymal focus and enlargement of regional intrathoracic nodes, especially in the hilum and mediastinum. ${ }^{20}$ Many of these children remain asymptomatic. The affected areas often heal, although the child will remain latently infected. Complications arise in a few children, usually because of the proximity of the lymph nodes to major airways. ${ }^{21}$ Enlargement of these nodes may lead to wheezing, segmental collapse or pneumonia related to airway compression. Uncommonly, the parenchymal focus will enlarge and cavitate; however, cavities are much more commonly seen in adolescent and adult TB. ${ }^{18}$

In North America, diagnosis of intrathoracic TB is often made when a child is evaluated because of contact with an infectious source case and is found to have an abnormal chest radiograph;22 these children often have few symptoms.

\section{How does TB disease present in adolescents?}

Adolescents may present with features seen in both children and adults. Fever, night sweats and weight loss are common symptoms. ${ }^{23}$ Pulmonary TB is the most common form of TB at this age and may present with cough and hemoptysis. Infiltrates and cavities may be seen on chest radiographs, but intrathoracic lymphadenopathy may also occur. ${ }^{23}$ Pleural disease may present as an acute febrile illness with pleuritic chest pain and nonproductive cough, ${ }^{24}$ and may be isolated or be associated with parenchymal abnormalities. ${ }^{23}$ Extrathoracic disease has been reported in $21 \%-37 \%$ of adolescents in low

Table 1: Interpretation of a tuberculin skin test ${ }^{10}$
Tuberculin reaction
( $\mathrm{mm}$ of induration)

$5-9$

$\geq 10$
Setting in which reaction is considered significant

HIV infection; contact with active contagious case; children with suspected TB disease; other immune suppression (e.g., treatment with TNF- $\alpha$ inhibitors or corticosteroids, chemotherapy, end-stage renal disease)

All other settings
Note: TNF = tumour necrosis factor. Administer test intradermally according to guidelines and read in $\mathrm{mm}$ of induration at 48-72 h. Test only those patients at risk for TB infection or those with progression of TB infection. Routine testing of individuals at low risk is not indicated. In young children with suspected TB disease, treatment may be started even if the tuberculin reaction result is $0-4 \mathrm{~mm}$ of induration. 
TB-burden settings. 1,23,25 Any organ system, including bones, joints and the central nervous system, may be involved. Peripheral lymphadenitis, often cervical, is the most common site of extrapulmonary TB and may present as painless swelling that sometimes occurs with constitutional symptoms. ${ }^{26,27}$ Presentation may mimic that of nontuberculous mycobacterial lymphadenitis, a syndrome that is more commonly seen in low TB-burden settings. ${ }^{28}$

Abdominal TB may mimic inflammatory bowel disease or present with ascites or a fibrotic involvement of the omentum and related structures. ${ }^{29}$

\section{How is TB diagnosed?}

Because of the myriad ways in which TB can present, TB disease should be suspected in any child with epidemiologic risk factors and suggestive symptoms or signs of organ involvement that are not explained by other diagnoses. Clinical suspicion is critical. Diagnosis is aided by diagnostic imaging, ancillary laboratory tests and by microbiologic confirmation. Positive results for tuberculin skin tests and interferon gamma release assays may support the diagnosis of TB disease, but results may be negative in as many as $30 \%$ of children with TB disease according to a meta-analysis published in $2011 .{ }^{13}$

\section{Diagnostic imaging}

A chest radiograph is diagnostically useful in children with suspected TB. In addition to the anterior posterior view, a lateral radiograph is helpful to assess hilar or mediastinal lymphadenopathy. ${ }^{30}$ Rotation of the child relative to the $x$-ray beam may lead to the false appearance of hilar enlargement: good positioning of the child and reading by a radiologist experienced in pulmonary TB are important. Chest radiography in adolescents often shows upper-lobe airspace disease, sometimes with cavitation. However, any lobe may be involved, and isolated lower lobe disease was found to occur in about $10 \%$ of cases in a retrospective review involving adolescents in Houston, Texas. ${ }^{23}$ Computed tomography (CT) may have characteristic findings of a "tree on bud" pattern, ${ }^{31}$ but CT is usually unnecessary: most pulmonary disease can be diagnosed with a combination of spontaneous or induced sputum culture and chest radiography.

For extrathoracic TB diagnosis, imaging should be performed according to symptoms and signs. Ultrasonography and CT aid diagnosis of abdominal and lymph node TB. Plain radiography and magnetic resonance imaging (MRI) are helpful for evaluation of osteoarticular TB. ${ }^{31}$ Magnetic resonance imaging has a wider range of soft tissue contrasts and shows more detailed central nervous system soft tissue anatomy than CT; thus, it is better at showing the extent of leptomeningeal enhancement and detecting parenchymal abnormalities characteristic of TB disease in the central nervous system. ${ }^{31}$ Hydrocephalus is common at presentation in TB meningitis. Contrast enhancement is needed to show the characteristic leptomeningeal enhancement that is often basilar.

\section{Laboratory tests and microbiologic confirmation}

During the last decade, over $9 \%$ of TB isolates in Canada were resistant to at least one first-line antituberculous agent. ${ }^{32}$ For this reason, diagnosis should be confirmed by obtaining specimens for microbiologic and drug-susceptibility testing. However, pediatric TB is often a paucibacillary disease: results for acid-fast bacillus stains are frequently negative and, despite best efforts, cultures may be negative for over $40 \%$ of children with TB disease. ${ }^{33}$ Diagnosis in children is often made on the basis of known contact with an infectious source case, a positive tuberculin skin test or interferon gamma release assay and characteristic abnormalities in chest radiography. After obtaining cultures, children with a high pretest probability of TB disease require treatment even if the cultures are negative; this especially applies to suspected TB meningitis, because serious complications, such as stroke, may develop rapidly. ${ }^{19}$

In adolescents with pulmonary disease, spontaneously expectorated sputum for TB culture should be obtained; however, induced sputum using $3 \%$ or $5 \%$ normal saline may increase culture yields by over $30 \%$ compared with spontaneous sputum ${ }^{34}$ and is useful in those unable to expectorate. ${ }^{35}$

In young children who cannot produce a sputum sample, early morning gastric aspirates are often used to obtain cultures in those with intrathoracic disease. ${ }^{36} \mathrm{~A}$ useful video resource is referenced in Box 3. Gastric aspirates should be buffered rapidly to improve culture yields. Three aspirates are recommended: in a recent retrospective study in Canada, the first aspirate identified $67 \%$, the second a further $20 \%$ and the third a further $13 \%$ of cases. ${ }^{37}$ For younger children, nebulization of hypertonic saline followed by a timed nasopharyngeal aspirate has yields similar to gastric aspirates in highburden settings ${ }^{38}$ and has been used in Canada as a supplement or alternative to gastric aspirates. In instances where there is one potential source case, the pattern of drug susceptibility in an adult contact may be used to guide empiric treatment in the child.

For those with extrapulmonary disease, it is important to send fresh biopsy tissue for mycobacterial culture. Pus swabs are unsuitable for TB culture.

Nucleic acid amplification tests, including polymerase chain reaction (PCR), allow faster diagnosis. Line probe assays provide rapid identification of the presence of TB and a preliminary identification of some drug susceptibilities. ${ }^{39}$ These results should be confirmed using analysis of phenotypic sensitivities.

Pleural biopsy has a higher yield than fluid culture. ${ }^{24}$ Unlike the pleural effusions associated with bacterial pneumonia, tuberculous pleural effusions often have lymphocyte predominance in cell counts. However, neutrophil predominance can occur in some cases. ${ }^{24}$

Cerebrospinal fluid in TB meningitis often has high protein, low glucose and lymphocyte predominance in cell counts; however, neutrophil predominance may occur. ${ }^{19}$ Results for cerebrospinal fluid culture are positive for TB meningitis in only $30 \%-35 \%$ of

\section{Box 3: Physician resources}

- Canadian Tuberculosis Standards (7th ed.; available at www.phac -aspc.gc.ca/tbpc-latb/pubs/tb-canada-7/)

- Curry International Tuberculosis Centre: Pediatric tuberculosis: a guide to the gastric aspirate (GA) procedure (available at www.currytbcenter.ucsf.edu/products/view/pediatric -tuberculosis-guide-gastric-aspirate-ga-procedure)

- Peel Public Health: Preventing tuberculosis (TB) disease in children (available at www.peelregion.ca/health/tb/other-languages.htm) 
cases, and results for acid-fast bacillus stains are almost always negative: a PCR test should also be requested. ${ }^{19}$

Tuberculin skin tests and interferon gamma release assays may provide supportive information, but they do not differentiate between infection and disease, and may give a negative result in many children with TB disease. ${ }^{13}$

Delay in diagnosis of TB in adolescents despite several physician visits has been reported. ${ }^{1}$ Some modifiable reasons for this delay from the authors' experience and the literature are shown in Box 4.

\section{How should latent TB infection be treated?}

About 5\%-10\% patients with latent TB are at risk of acquiring active TB disease over their lifetime, but up to $50 \%$ of children younger than one year of age will acquire TB disease. ${ }^{16}$ Immunosuppression (including HIV infection, transplantation, renal failure, corticosteroid and anti-tumour necrosis factor- $\alpha$ treatment) and recent tuberculin skin test conversion also substantially increase this risk of progression. ${ }^{40}$ Randomized trials conducted in an era of isoniazid-sensitive disease showed that treatment with isoniazid

Box 4: Missed opportunities for early diagnosis of pediatric tuberculosis in Canada ${ }^{1}$

- Failure to consider epidemiologic history

- Failure to obtain sputum for TB culture

- Failure to send biopsy specimens for TB culture

- Failure to consider a diagnosis of TB, because of a negative result for the tuberculin skin test

- Failure to consider TB in the following circumstances:

- Poorly responding pneumonia

- Pleural effusion with or without parenchymal change

- Hydrocephalus and meningitis with negative results for bacterial cultures

- Prolonged fever in the returning traveller

\section{Box 5: Management of pediatric tuberculosis disease}

Tests that should be considered

- Posteroanterior and lateral chest radiographs

- Other imaging as clinically indicated

- Tuberculosis cultures (without formalin) as indicated: sputum (induced if possible), gastric aspirates, biopsies, cerebrospinal fluid; liaise with TB laboratory

- Tuberculin skin test/interferon gamma release assay: test result may be negative in $>10 \%$ of patients; if test result is positive, consider it supportive evidence

\section{How to start management of TB disease}

- Ensure appropriate isolation

- Notify and involve local public health unit

- Involve TB team and experts

- See Canadian Tuberculosis Standards for details ${ }^{3}$

Adapted from Paediatr Child Health 2015;20:83-8. ${ }^{4}$ for nine months is highly protective and reduces the risk of acquiring TB in a lifetime by over $80 \% .{ }^{41}$ Isoniazid hepatotoxicity in children is uncommon but can occur and may lead to liver transplantation and death. ${ }^{42}$ Patients and their parents should be informed about the risks and benefits of treatment for latent disease and encouraged to stop treatment with isoniazid if anorexia, nausea, jaundice or vomiting occur. ${ }^{43}$ Should such symptoms develop, levels of serum transaminases and bilirubin should be checked.

Patients should receive a written plan of action in the language they most prefer that includes contact numbers if adverse effects occur or questions arise. Patients receiving isoniazid should be seen at least once a month to monitor for adverse effects. Blood tests are not routinely recommended if the patient is well but should be performed if any symptoms occur. For those patients who acquire infection after contact with a drug-resistant strain, expert opinion is recommended.

\section{What are the general principles of treating TB disease?}

Best results are achieved if treatment is conducted by or in conjunction with a team comprising clinicians, public health nurses, translation services and social work support that is expert in the management of TB disease in childhood (Box 5). ${ }^{3}$ A retrospective population-based study in Ontario found that directly observed therapy, in which a health care worker observes administration of medication, improved survival rates for $\mathrm{TB},{ }^{2}$ and it is the recommended approach for treatment of pediatric patients with TB disease. ${ }^{3}$ Physician experience in treating TB and language concordance between patient and health care provider have been shown to affect outcome:2,44 translation services (which can be telephone based) should be used whenever there is any difficulty with language.

Optimal treatment for TB disease includes multiple drugs to prevent the emergence of resistant strains (Appendix 1, available at www.cmaj.ca/lookup/suppl/doi:10.1503/cmaj.151212/-/DC1). The mode and mechanism of action of each drug, dosages in children and main toxicities are shown in Appendix 1 and may be found in Canadian Tuberculosis Standards. ${ }^{3}$

\section{How should childhood contacts of patients with TB be managed?}

Management of close contacts is a critical part of TB prevention, and Figure 1 outlines an approach based on the Canadian Tuberculosis Standards. ${ }^{3}$ Children and adolescents exposed to infectious TB should have a tuberculin skin test and a chest radiograph. If the initial tuberculin skin test result is negative, it should be repeated 8-10 weeks after the last date the child had contact with an infectious source case (the "break in contact" date). Those patients who have a positive tuberculin skin test result and have no clinical or radiologic evidence of disease should be treated for latent TB infection based on source case sensitivities. Because young contacts are at high risk for rapid development of serious forms of TB, children younger than five years of age with a negative result for the tuberculin skin test are treated with a regi- 


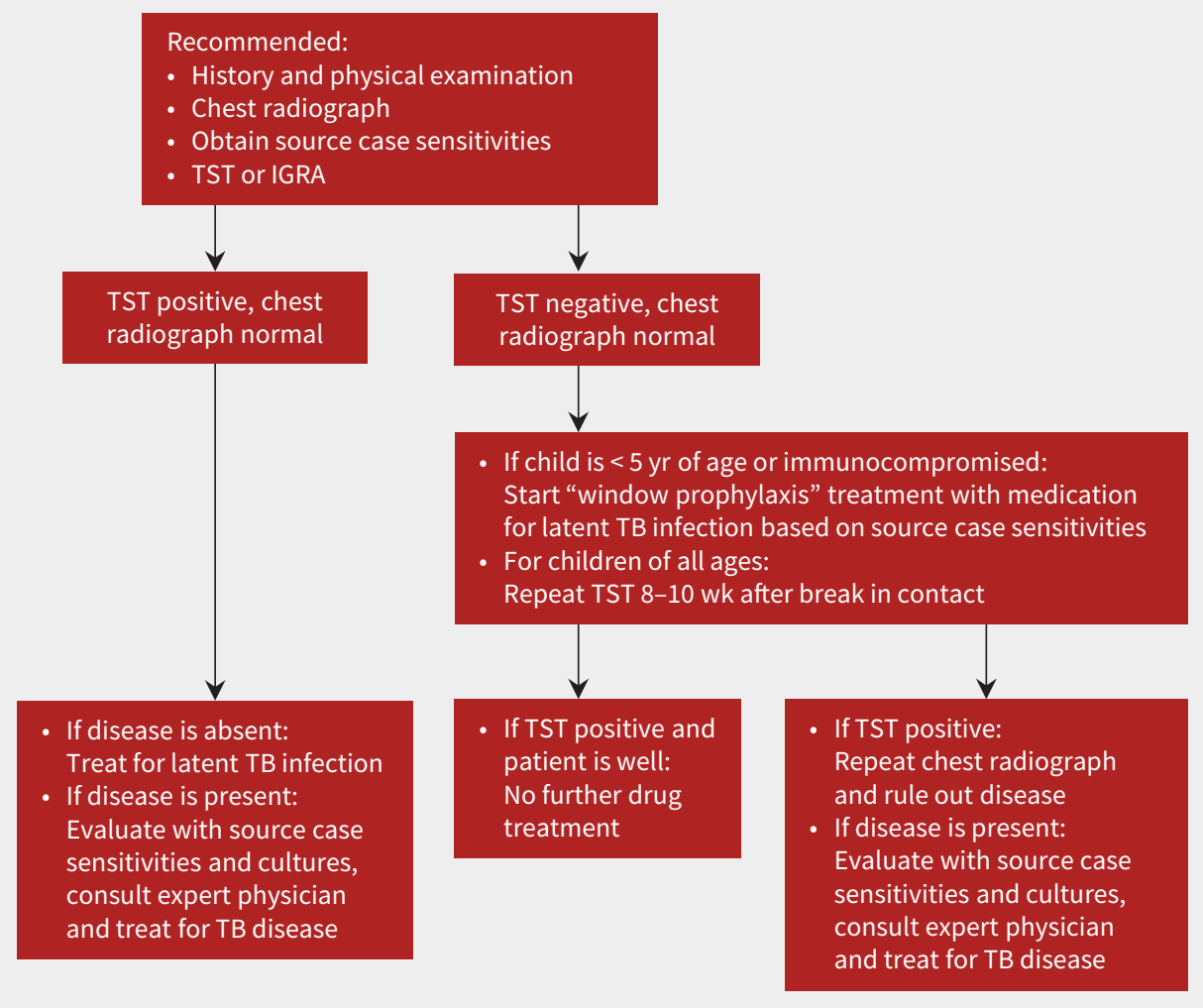

Figure 1: Management of treatment in children exposed to infectious tuberculosis (TB). IGRA = interferon gamma release assay, TST = tuberculin skin test. Adapted from Paediatr Child Health 2015;20:83-8. ${ }^{4}$

men effective against latent TB infection until the second tuberculin skin test result is known to be negative: this is referred to as window prophylaxis. ${ }^{3}$

\section{How can TB in children be prevented in Canada?}

Optimal management of patients who are in contact with an infectious source case will prevent disease in children and prevent them from becoming infectious adults. Early diagnosis of TB disease in adolescents may reduce the length of time that they are infectious. Screening for latent TB disease in children who are immigrating to Canada or being adopted from countries where TB is endemic has been recommended. ${ }^{45,46}$ However, this routine approach to detection and treatment in this group, although important for elimination of TB in North America, ${ }^{9}$ also requires careful monitoring for toxicity and a frank discussion of risks and benefits. Shorter-course regimens for latent TB infection, including the treatment combination of isoniazid and rifapentine taken once weekly for three months (not currently available in Canada except under study conditions), ${ }^{47}$ hold promise for better sustainability and better rates of treatment completion. Interferon gamma release assay may be preferable to the tuberculin skin test for screening children older than five years of age who were given the bacille Calmette-Guérin vaccine and are at a lower risk, and allows more targeted treatment. ${ }^{12,48}$

High rates of TB among the Inuit and other Aboriginal groups in Canada deserve much greater national focus and will require coordinated public health measures and innovative programs ${ }^{49}$ but also substantial improvement in housing and living conditions. ${ }^{50}$

Finally, elimination of TB in Canada also depends on a global effort to control and eliminate TB. It is in Canada's interests to participate actively in this international effort.

\section{References}

1. Kam A, Ford-Jones L, Malloy P, et al. Active tuberculosis among adolescents in Toronto, Canada: clinical features and delays in diagnosis. Pediatr Infect Dis $J$ 2007;26:355-6.

2. Khan K, Campbell A, Wallington $\mathrm{T}$, et al. The impact of physician training and experience on the survival of patients with active tuberculosis. CMAJ 2006;175: 749-53.

3. Kitai I, Demers AM. Paediatric tuberculosis. Chapter 9. In: Menzies D, ed. Canadian Tuberculosis Standards. 7th ed. Ottawa: Public Health Agency of Canada; 2013. Available: www.phac-aspc.gc.ca/tbpc-latb/pubs/tb-canada-7/tb-standards-tb -normes-ch9-eng.php (accessed 2015 Oct. 8).

4. Morris SK, Demers AM, Lam R, et al. Epidemiology and clinical management of tuberculosis in children in Canada. Paediatr Child Health 2015;20:83-8.

5. Halverson J, Ellis E, Gallant V, et al. Epidemiology of tuberculosis in Canada. Chapter 1. In: Menzies D, ed. Canadian Tuberculosis Standards. 7th ed. Ottawa: Public Health Agency of Canada; 2014. Available: www.phac-aspc.gc.ca/tbpc-latb/pubs/ tb-canada-7/tb-standards-tb-normes-ch1-eng.php (accessed 2015 Oct. 8). 
6. Tuberculosis in Canada 2014 - Pre-release. Ottawa: Public Health Agency of Canada; 2016. Available: http://healthycanadians.gc.ca/publications/diseases -conditions-maladies-affections/tuberculosis-2014-tuberculose/index-eng.php (accessed 2015 Oct. 8).

7. Rayment JH, Guthrie JL, Lee B, et al. Culture-positive paediatric tuberculosis in Toronto, Ontario: sources of infection and relationship of birthplace and mycobacterial lineage to phenotype. Pediatr Infect Dis J 2016;35:13-8.

8. Winston CA, Menzies HJ. Pediatric and adolescent tuberculosis in the United States, 2008-2010. Pediatrics 2012;130:e1425-32.

9. Horsburgh CR Jr, Rubin EJ. Clinical practice. Latent tuberculosis infection in the United States. N Engl J Med 2011;364:1441-8.

10. Pai M, Kunimoto D, Jamieson F, et al. Diagnosis of latent tuberculosis infec tion. Chapter 4. Canadian Tuberculosis Standards. 7th ed. Ottawa: Public Health Agency of Canada; 2013. Available: www.phac-aspc.gc.ca/tbpc-latb/pubs/tb-canada-7/tb -standards-tb-normes-ch4-eng.php (accessed 2015 Oct. 8).

11. Starke JR; Committee On Infectious Diseases. Interferon-gamma release assays for diagnosis of tuberculosis infection and disease in children. Pediatrics 2014; 134:e1763-73.

12. Rose W, Read SE, Bitnun A, et al. Relating tuberculosis (TB) contact characteristics to QuantiFERON-TB-Gold and tuberculin skin test results in the Toronto Pediatric TB Clinic. J Pediatric Infect Dis Soc 2015;4:96-103.

13. Machingaidze S, Wiysonge CS, Gonzalez-Angulo Y, et al. The utility of an interferon gamma release assay for diagnosis of latent tuberculosis infection and disease in children: a systematic review and meta-analysis. Pediatr Infect Dis J 2011;30:694-700.

14. Pai M, Denkinger CM, Kik SV, et al. Gamma interferon release assays for detection of Mycobacterium tuberculosis infection. Clin Microbiol Rev 2014;27:3-20.

15. Seddon JA, Shingadia D. Epidemiology and disease burden of tuberculosis in children: a global perspective. Infect Drug Resist 2014;7:153-65

16. Marais BJ, Gie RP, Schaaf HS, et al. The natural history of childhood intra-thoracic tuberculosis: a critical review of literature from the pre-chemotherapy era. Int J Tuberc Lung Dis 2004;8:392-402.

17. Graham SM, Ahmed T, Amanullah F, et al. Evaluation of tuberculosis diagnostics in children: 1 . Proposed clinical case definitions for classification of intrathoracic tuberculosis disease. Consensus from an expert panel. $J$ Infect Dis 2012; 205(Suppl 2):S199-208.

18. Hesseling AC, Schaaf HS, Gie RP, et al. A critical review of diagnostic approaches used in the diagnosis of childhood tuberculosis. Int J Tuberc Lung Dis 2002; 6:1038-45.

19. van Toorn R, Solomons R. Update on the diagnosis and management of tuberculous meningitis in children. Semin Pediatr Neurol 2014;21:12-8.

20. Marais BJ, Gie RP, Schaaf HS, et al. A proposed radiological classification of childhood intra-thoracic tuberculosis. Pediatr Radiol 2004;34:886-94.

21. Cruz AT, Starke JR. Clinical manifestations of tuberculosis in children. Paediatr Respir Rev 2007;8:107-17.

22. Starke JR. Diagnosis of tuberculosis in children. Pediatr Infect Dis J 2000;19:1095-6

23. Cruz AT, Hwang KM, Birnbaum GD, et al. Adolescents with tuberculosis: a review of 145 cases. Pediatr Infect Dis J 2013;32:937-41.

24. Vorster MJ, Allwood BW, Diacon AH, et al. Tuberculous pleural effusions: advances and controversies. J Thorac Dis 2015;7:981-91.

25. Phongsamart W, Kitai I, Gardam M, et al. A population-based study of tuberculosis in children and adolescents in Ontario. Pediatr Infect Dis J 2009;28:416-9.

26. Fontanilla JM, Barnes A, von Reyn CF. Current diagnosis and management of peripheral tuberculous lymphadenitis. Clin Infect Dis 2011;53:555-62.

27. Donald PR. The chemotherapy of tuberculous lymphadenopathy in children. Tuberculosis (Edinb) 2010;90:213-24

28. Tebruegge M, Pantazidou A, MacGregor D, et al. Nontuberculous mycobacterial disease in children - Epidemiology, diagnosis \& management at a tertiary center. PLoS One 2016;11:e0147513.

29. Debi U, Ravisankar V, Prasad KK, et al. Abdominal tuberculosis of the gastrointestinal tract: revisited. World J Gastroenterol 2014;20:14831-40.
30. Andronikou S, Vanhoenacker FM, De Backer Al. Advances in imaging chest tuberculosis: blurring of differences between children and adults. Clin Chest Med 2009;30:717-44.

31. Skoura E, Zumla A, Bomanji J. Imaging in tuberculosis. Int J Infect Dis 2015;32: 87-93.

32. Canadian Tuberculosis Standards. 7th ed. Ottawa: Public Health Agency of Canada; 2014. Available: www.phac-aspc.gc.ca/tbpc-latb/pubs/tb-canada-7 (accessed 2015 Oct. 8)

33. Thampi N, Stephens D, Rea E, et al. Unexplained deterioration during antituberculous therapy in children and adolescents: clinical presentation and risk factors. Pediatr Infect Dis J 2012;31:129-33.

34. Schoch OD, Rieder P, Tueller C, et al. Diagnostic yield of sputum, induced sputum, and bronchoscopy after radiologic tuberculosis screening. Am J Respir Crit Care Med 2007;175:80-6.

35. Brown $\mathrm{M}$, Varia $\mathrm{H}$, Bassett $\mathrm{P}$, et al. Prospective study of sputum induction, gastric washing, and bronchoalveolar lavage for the diagnosis of pulmonary tuberculosis in patients who are unable to expectorate. Clin Infect Dis 2007;44:1415-20.

36. Cruz AT, Revell PA, Starke JR. Gastric aspirate yield for children with suspected pulmonary tuberculosis. J Pediatric Infect Dis Soc 2013;2:171-4.

37. Kordy F, Richardson SE, Stephens D, et al. Utility of gastric aspirates for diagnosing tuberculosis in children in a low prevalence area: predictors of positive cultures and significance of non-tuberculous mycobacteria. Pediatr Infect Dis J 2015; 34:91-3.

38. Zar HJ, Hanslo D, Apolles P, et al. Induced sputum versus gastric lavage for microbiological confirmation of pulmonary tuberculosis in infants and young children: a prospective study. Lancet 2005;365:130-4.

39. Drobniewski F, Cooke M, Jordan J, et al. Systematic review, meta-analysis and economic modelling of molecular diagnostic tests for antibiotic resistance in tuberculosis. Health Technol Assess 2015;19:1-188.

40. Menzies D, Alvarez GG, Khan K. Treatment of latent tuberculosis infection. Chapter 6 . Canadian Tuberculosis Standards. 7th ed. Ottawa: Public Health Agency of Canada; 2013. Available: www.phac-aspc.gc.ca/tbpc-latb/pubs/tb-canada-7/tb-standards-tb -normes-ch6-eng.php (accessed 2015 Oct. 8).

41. Ferebee $\mathrm{SH}$. Controlled chemoprophylaxis trials in tuberculosis. A general review. Bibl Tuberc 1970;26:28-106.

42. Centers for Disease Control and Prevention (CDC). Severe isoniazid-associated liver injuries among persons being treated for latent tuberculosis infection United States, 2004-2008. MMWR Morb Mortal Wkly Rep 2010;59:224-9.

43. Science M, Ito S, Kitai I. Isoniazid toxicity in a 5-year-old boy. CMAJ 2013;185:894-6.

44. Gardam M, Verma G, Campbell A, et al. Impact of the patient-provider relationship on the survival of foreign born outpatients with tuberculosis. J Immigr Minor Health 2009;11:437-45.

45. Greenaway C, Sandoe A, Vissandjee B, et al.; Canadian Collaboration for Immigrant and Refugee Health. Tuberculosis: evidence review for newly arriving immigrants and refugees. CMAJ 2011;183:E939-51.

46. Long R, Boffa J. Why internationally adopted children should be screened for tuberculosis. CMAJ 2007;177:172-3.

47. Villarino ME, Scott NA, Weis SE, et al.; International Maternal Pediatric and Adolescents AIDS Clinical Trials Group. Tuberculosis Trials Consortium. Treatment for preventing tuberculosis in children and adolescents: a randomized clinical trial of a 3-month, 12-dose regimen of a combination of rifapentine and isoniazid. JAMA Pediatr 2015;169:247-55.

48. Ling DI, Crépeau CA, Dufresne M, et al. Evaluation of the impact of interferongamma release assays on the management of childhood tuberculosis. Pediatr Infect Dis J 2012;31:1258-62.

49. Alvarez GG, VanDyk DD, Aaron SD, et al. Taima (stop) TB: the impact of a multifaceted TB awareness and door-to-door campaign in residential areas of high risk for TB in Iqaluit, Nunavut. PLoS One 2014;9:e100975.

50. MacDonald N, Hébert PC, Stanbrook MB. Tuberculosis in Nunavut: a century of failure. CMAJ 2011;183:741-3.

\section{Competing interests: None declared.}

This article was solicited and has been peer reviewed.

Affiliations: Division of Infectious Diseases (Kitai, Morris, Kordy, Lam); Centre for Global Child Health (Morris), Hospital for Sick Children; Department of Pediatrics (Kitai, Morris); Faculty of Nursing (Lam), University of Toronto, Toronto, Ont.
Contributors: Ian Kitai conducted the original literature search and drafted the first version of the article. All of the authors assisted with the literature search and retrieval of articles. Shaun Morris, Faisal Kordy and Ray Lam revised the manuscript critically for important intellectual content. All of the authors approved the final version to be published and agreed to act as guarantors of the work.

Correspondence to: Ian Kitai, ian.kitai@sickkids.ca 